\title{
Special Issue: International Conference on Applied System Innovation (ICASI 2017)
}

\author{
Sheng-Joue Young ${ }^{1} \cdot$ Teen-Hang Meen ${ }^{1} \cdot$ Ajit Khosla $^{2} \cdot$ B. Michel ${ }^{3}$
}

Published online: 11 May 2018

(C) Springer-Verlag GmbH Germany, part of Springer Nature 2018

The IEEE International Conference on Applied System Innovation (ICASI) 2017 was held successfully on May 13-17, 2017, at Sapporo, Japan. ICASI provides a communication bridge for researchers worldwide and enables international and interdisciplinary collaborations between science and engineering technologists in the academic and industrial fields, as well as in networking fields. ICASI 2017 covered a wide range of topics, including information science, innovation design, industrial design, applied mathematics, computer science, cultural and creative research, electrical and electronic engineering, mechanical engineering, automation engineering, green technology and architecture engineering, material science, and other related fields.

This special issue presents 53 excellent papers selected from ICASI 2017 that cover a wide range of fundamental studies, industrial practices, and engineering innovations by applying knowledge on microsystems. The Internet of things (IOT) has also gained popularity in recent years. In fact, this special issue collected many IOT-related studies, such as those on sensors, electronics, software, and connectivity, which enable objects to connect and exchange data.

\footnotetext{
Ajit Khosla

khosla@gmail.com

Sheng-Joue Young

shengjoueyoung@gmail.com

Teen-Hang Meen

thmeen@nfu.edu.tw

B. Michel

bernd.michel@coinn.de

1 Department of Electronic Engineering, National Formosa University, Huwei 632, Yunlin, Taiwan

2 Faculty of Engineering, Yamagata University, 4-3-16 Jonan, Yonezawa, Yamagata 992-8510, Japan

3 Fraunhofer Micro Materials Center Berlin, Berlin, Germany
}

All articles in this issue underwent rigorous peer reviews conducted by Microsystem Technologies. The acceptance of this special issue was based on recommendations of the reviewers, mandatory revisions, final examinations, and reviews conducted by the guest editors. This publication is expected to be a key issue for Microsystem Technologies.

The guest editors would like to thank the authors for their contributions and all the reviewers for their constructive comments. Moreover, we would like to give special thanks to the Editor-in-Chief, Prof. Bernd Michel, for his generous support in the publication of this issue. Finally, we would like to thank the editorial team for their work in making this special issue a success.

We look forward to your participation in future special issues of Applied System Innovation.

Sincerely,

Guest editors

Prof. Sheng-Joue Young

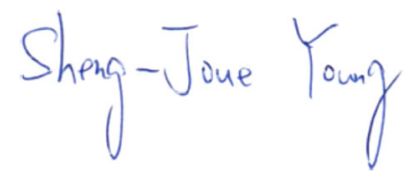

Prof. Teen-Hang Meen

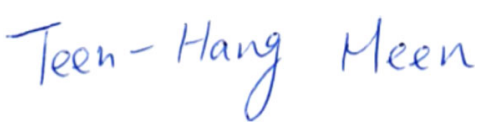

Prof. Ajit Khosla

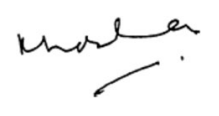

Prof. B. Michel (Editor-in-Chief) 